\section{EL AMOR, EL DESTINO Y LA VIRTUD: GERTRUDIS GÓMEZ DE AVELLANEDA Y SU PRIMER DRAMA LEONCIA}

\author{
Alexander Selimov \\ University of Delaware \\ ale@udel.edu
}

Cómo citar este artículo/Citation: Selimov, A. (2014). "EI amor, el destino y la virtud: Gertrudis Gómez de Avellaneda y su primer drama Leoncia". Arbor, 190 (770): a187. doi: http:// dx.doi.org/10.3989/arbor.2014.770n6008

Recibido: 12 febrero 2014. Aceptado: 30 octubre 2014.

RESUMEN: El éxito del drama romántico Leoncia marca el comienzo de la trayectoria profesional de Gertrudis Gómez de Avellaneda en el ámbito teatral. Los críticos literarios han visto en esta obra la elaboración de dos grandes tópicos románticos, el amor y el destino. En el presente ensayo se argumenta que la autora se sirve de la retórica romántica para abordar el tema de la virtud, exponiendo su manipulación dentro de la estructura jerárquica de subordinación en la sociedad patriarcal. La preocupación en torno al concepto de la virtud permea gran parte de la obra de la autora hispano-cubana y a pesar de que su discurso se presenta contaminado por la retórica patriarcal, es indicativo de su esfuerzo por trascender el papel subordinado en la jerarquía social de la España decimonónica.

PALABRAS CLAVE: Gómez de Avellaneda; el drama romántico; amor; destino; virtud; patriarcado; subalternidad; feminismo.

\section{LOVE, DESTINY AND VIRTUE: GERTRUDIS GÓMEZ DE AVELLANEDA AND HER FIRST DRAMA LEONCIA}

Copyright: (C) 2014 CSIC. Este es un artículo de acceso abierto distribuido bajo los términos de la licencia Creative Commons Attribution-Non Commercial (by-nc) Spain 3.0.
ABSTRACT: The success of the romantic drama Leoncia marks the beginning of Gertrudis Gómez de Avellaneda's career in the theatre. Literary critics have seen in this work two great romantic topics, love and destiny. This essay argues that the author uses romantic rhetoric to address the issue of virtue and of its manipulation within a hierarchical structure of subordination in a patriarchal society. This concern with the concept of virtue permeates much of the work of this SpanishCuban author, and is part of her pioneering efforts to expose the problem of women's subordinate role in the social hierarchy in nineteenth-century Spain.
KEYWORDS: Gómez de Avellaneda; romantic drama; love; destiny; virtue; patriarchy; subalternity; feminism. 
En junio de 1840 se estrena en Sevilla un drama romántico de asunto contemporáneo titulado Leoncia. Su autora, Gertrudis Gómez de Avellaneda (18141873) llegó a la ciudad el año anterior, procedente de la Coruña, donde residía con su madre y padrastro luego de su arribo de Cuba en 1836. El público recibió con aplausos la primera pieza trágica de la dramaturga debutante, a quien para entonces solo conocía por la publicación de algunos poemas originales y traducciones de la poesía de Lamartine y Millevoye (Gómez de Avellaneda, 1999, p. 92). El exitoso estreno de Leoncia estimuló a la autora a perseverar en su empeño, marcando el comienzo de lo que sería una gran trayectoria profesional.

Entre 1840 y 1844, Gertrudis Gómez publica un volumen de poesía y varias novelas, al mismo tiempo que continúa escribiendo para el teatro, el campo "donde tantas victorias le aguardaban" (Piñeyro, 1904, p. 152). Entre 1844 y 1859 envía a las tablas unas diecisiete piezas, incluyendo cinco obras trágicas originales: Alfonso Munio, El príncipe de Viana, Egilona, Saúl y Baltazar. Todas las tragedias fueron aplaudidas en sus estrenos por los contemporáneos, pero las que obtuvieron una recepción más favorable entre el público son la primera y la última de la lista. Alfonso Munio, en particular, gozó de un éxito abrumador y le granjeó a la autora el reconocimiento inmediato de los círculos literarios capitalinos, mientras que Baltazar llegó a ser considerado una de las obras dramáticas cumbre del siglo XIX español.

A juzgar por los primeros comentarios críticos, Leoncia impresionó a los críticos de teatro, y aunque no llegó a representarse en Madrid, e incluso quedó inédita durante el siglo XIX, logró poner el nombre de su autora en la cartelera y crear una expectativa de continuidad entre los críticos y los aficionados al teatro, como pone en evidencia la reseña de la obra aparecida en la revista La Alhambra de Granada el ocho de noviembre de 1840 :

Un drama, primera composición de una joven española [...] debía excitar y excitó vivamente nuestra curiosidad. Es tan raro el número de producciones originales que se representan en el teatro [...]. Presentíamos y no nos engañábamos que la Leoncia sería un drama de pasión, y teníamos curiosidad de ver cómo la trataba en el extenso cuadro que se había propuesto [...] ¿Y qué le diremos a nuestros lectores? Que La Peregrina ha triunfado en la empresa que se propuso [...] Hemos visto a un público, bien difícil de contentar por cierto, escuchar con una atención sostenida los últimos actos del drama, y hemos sor- prendido el llanto en los ojos de personas demasiado afligidas por los sucesos reales de la vida para derramarlas si no fuera por un poderoso interés (citado por Cotarelo, 1930, pp. 425-426).

No es de extrañar que la primera obra teatral de la joven Avellaneda tuviera una recepción crítica tan entusiasta. A pesar del triunfo definitivo de la estética romántica frente al neoclasicismo en el ámbito teatral nacional, logrado por los autores como Duque de Rivas, García Gutiérrez, Hartzenbusch, Larra y Martínez de la Rosa, la escena española seguía dominada por la opera, el melodrama francés y las refundiciones de las obras del Siglo de Oro (Gies, 1996, p. 19). Con Leoncia, Gómez de Avellaneda le ofreció al público un drama original que por su acción dinámica, situaciones dramáticas, un conflicto polarizado y un final patético y espectacular, se acomodaba perfectamente al gusto romántico imperante en la época. Si se considera el hecho que la participación de las mujeres en la producción teatral se limitaba tradicionalmente a una función subordinada, de actriz o costurera, es justo afirmar que el éxito de Gertrudis Gómez inspiró a otras autoras españolas a abrirse paso en el campo de dramaturgia a partir de mediados del siglo diecinueve (Gies, 1996, pp. 268-269).

El argumento de Leoncia se estructura en torno a un triángulo amoroso conformado por tres figurantes, don Carlos Maldonado, su novia doña Elena de Castro, y Leoncia, una bella y misteriosa mujer madura, hacia quien Carlos siente una atracción fatal. Los rumores sobre una supuesta relación amorosa entre Leoncia, a quien solo se conoce por su nombre de pila, y su protector, el anciano conde Peñafiel, complican aún más la situación. La intriga se desarrolla de acuerdo al modelo romántico, es decir, entre el individuo desgraciado y la sociedad corrompida. Leoncia posee un alma virtuosa pero ha sufrido una experiencia traumática en su juventud: fue violada por cierto calavera sin nombre y tuvo una hija como consecuencia del desafortunado incidente. Para colmo de males, la pequeña desaparece en un naufragio junto con la madre de Leoncia. Con la ayuda del conde de Peñafiel, quien trata a Leoncia en todo momento como si fuera su hija, la dama logra alguna estabilidad en su vida, pero no puede vencer los prejuicios sociales, y al llegar a Madrid se convierte en objeto de chismes y rumores malintencionados:

DON GASPAR: (sonriendo con malicia.) Que no se sepa quién es ni de dónde ha venido. Una que, en dos años que hace está en Madrid, se haya rodeado de tal misterio, que ni sus mayores amigos hayan podido 
deducir si es rica o pobre, noble o plebeya, viuda o soltera, española o extranjera. Una que sea un enigma en la sociedad, que, cansada de querer en vano comprenderla, ha concluido por despreciarla (Gómez de Avellaneda, 1917, pp. 4-5).

Sintiéndose devastada y ultrajada por su pasado, "marcada por la sociedad con el sello de reprobación" (Cotarelo, 1930, p. 426), Leoncia sufre la conciencia de la imposibilidad de unirse a Carlos en matrimonio. Este último suplica a su amada que le revele los detalles sobre su pasado, pero la dama evade sus preguntas por vergüenza y temor de un rechazo. Prefiere sumergirse en el mundo de sus ilusiones enfocando el amor totalizador que los une a los dos. La conversación se interrumpe por la llegada del conde Peñafiel, lo cual enfurece y decepciona a Carlos, ya que le hace creer confirmados los rumores sobre la conducta indiscreta de Leoncia. La culminación del conflicto y la solución ocurren cuando la dama recibe la noticia de que su amado, frustrado por celos y despecho, decide cumplir el compromiso de matrimonio contraído con Elena. En un arrebato emocional, Leoncia se disfraza de modista y armada de un puñal va a la casa de la joven novia, supuestamente para suicidarse delante de la pareja. Una vez dentro de la casa, sorpresivamente descubre en Elena a su hija perdida, a quien creía muerta. Momentos después llega a la casa de novios el padre de Carlos, Don Fernando, en quien Leoncia reconoce al calavera, causante de todas sus desgracias, y se lanza sobre él con el puñal. Al interponerse Elena entre ellos, vuelve la daga contra su pecho y se suicida exclamando que Carlos y Elena son hermanos.

La estructura de la pieza contiene elementos típicos de la técnica romántica: un lenguaje patético y exaltado, caracterizado por un exagerado expresionismo sentimental, la presencia de una gran pasión que da sentido y razón a la existencia de los personajes y determina su comportamiento, el protagonismo de un alma superior en conflicto con una sociedad hostil, y finalmente, la influencia de aquella fuerza fatal del destino que baraja y dispone los eventos con una ironía macabra. La intriga es dinámica y bien construida, aun cuando en algunos momentos se desliza hacia la dimensión melodramática, y en otros, parece desafiar el criterio de la verosimilitud. La protagonista central es víctima de una pasión avasalladora, y según la opinión de los primeros estudiosos de la obra de Gertrudis Gómez, es "un personaje colosal, en quien (se) desenvuelve esta pasión de un modo admirable" (Gómez de Avellaneda, 1917, pp. 4-5). El tema del amor tiene una identidad romántica en tanto que identifica la fuente primaria del sentimiento con el proceso de ideación neoplásica en el contexto de una realidad ilusionada. Se trata del producto de las ilusiones y deseos de la primera juventud, el que se proyecta sobre un ser ideal imaginado: "es el amor que al mismo amor adora / el que creó las sílfides y ondinas, / la sacra ninfa que bordando mora / debajo de las aguas cristalinas" (Espronceda, 1978, p. 228). En 1840, Gertrudis Gómez de Avellaneda por boca de Leoncia declara: "La actividad naciente del corazón, que busca un objeto en quien complacerse; la necesidad de amar...: jéste es el origen del primer amor!” (Gómez de Avellaneda, 1917, p. 12), y en 1861 escribe ya con convicción de madurez que "se ama al amor y no al amante, según ha dicho no sé que filosofo. Se ama la propia facultad de amar, que comenzamos a sentir en nosotros" (Gómez de Avellaneda, 1990, p. 161). Es decir, a diferencia de la visión estética dominante en la época anterior, de origen renacentista, la visión romántica desvincula el sentimiento amoroso de la experiencia empírica, asociándolo con la necesidad natural de exteriorizar una subjetividad sensible: el amor no llega por contagio, hechizo o embrujo, sino que nace en el corazón del individuo. Por otra parte, el amor romántico siempre lleva un sello trágico de la muerte o desilusión, precisamente, por encarnar el ideal "que embelleció los sueños de la primera juventud" (Gómez de Avellaneda, 1917, p. 13), un ideal desconectado de la realidad y destinado al fracaso como consecuencia del proceso de conocimiento, tan bien ilustrado en el Canto a Teresa de José de Espronceda (Espronceda, 1978, p. 228).

Se ha dicho que el tema principal de Leoncia es el amor romántico, o más precisamente, "la tragedia de una pasión imposible e incumplida" (Prado, 2004, p. 159), y en efecto, en la pieza hay referencias a la identificación entre este y la razón de vivir de los amantes. Por ejemplo, en la escena VII del primer acto, Leoncia le recrimina a Carlos su demora con las siguientes palabras: "iCuánto he padecido con tu tardanza! Sólo existo cuando estoy junto a ti, y desde ayer, no te veo," a lo que el último le responde con sus propias quejas aceptando al final "que no podemos ya existir el uno sin el otro" (Gómez de Avellaneda, 1917, p. 13). Por su parte, Emilio Cotarelo ha sugerido que el tema de la tragedia era "el destino fatal e inexorable" (Cotarelo, 1930, p. 65), aquel gran tópico romántico, cuya presencia en la obra se hace patente al final del tercer acto con múltiples anagnórisis y el suicidio de la heroína que las sella. La funcionalidad esencial del amor y del destino en la estructura de la Leoncia consiste en matizar el estado de angustia y desesperación que 
los protagonistas sufren en su conflicto con el mundo, y al mismo tiempo coadyuva a crear la tensión y mantener al lector enganchado mientras la acción se desencadena hacia un final trágico. El destino y el amor pueden ser clasificados como catalizadores del ambiente romántico y de la acción dramática, lo cual los relega a un espacio secundario dentro de la arquitectura de la obra, mientras que el tema central está relacionado más bien con el concepto de la virtud. La tragicidad de la pieza está directamente relacionada con el conflicto entre el amor ideal y la hipocresía de una sociedad que a la usanza romántica aparece hostil e intolerante. Sin embargo, la clave del conflicto es su dimensión moral, ya que el obstáculo que les impide a Leoncia y Carlos lograr la realización de su amor no es una barrera de clase, religión o categoría social, sino una visión social de la virtud, o según se sugiere, una falsa percepción de la virtud.

Desde las primeras escenas de la pieza, se pone en evidencia la falta de congruencia entre los valores individuales del ser humano, identificados en la época ilustrada con la virtud absoluta, y las cualidades asociadas con la virtud relativa en una sociedad corrompida. Ser virtuoso en esta sociedad implica la obligación de mantener ciertas apariencias y "desgraciados aquellos que, apoyados en la rectitud de su corazón y en la pureza de su conciencia se atreven a infringir la más ligera de sus leyes!" -según se queja amargamente don Carlos (Gómez de Avellaneda, 1917, p. 7).

La preocupación ante la instrumentalización del concepto de la virtud en el sistema patriarcal para la reglamentación ética de la conducta humana es una constante en los textos públicos y privados de Gertrudis Gómez (Selimov, 1999, p. 222). Uno de los fragmentos más conocidos que la transmite se encuentra en su primera novela Sab:

Pero ¿qué es la virtud? ¿en qué consiste?... Yo he deseado comprenderlo, pero en vano he preguntado la verdad a los hombres. Me acuerdo que cuando mi amo me enviaba a confesar mis culpas a los pies de un sacerdote, yo preguntaba al ministro de Dios qué haría para alcanzar la virtud. La virtud del esclavo, me respondía, es obedecer y callar, servir con humildad y resignación a sus legítimos dueños, y no juzgarlos nunca. (Gómez de Avellaneda, 2001, p. 188).

El protagonista de la novela, luego de afrontar retóricamente las limitaciones del espacio que ocupa dentro de la jerarquía social cubana, sugiere un paralelo con el papel asignado a las mujeres en la sociedad: "¿No oís una voz, Teresa? Es la de los fuertes que dice a los débiles: 'Obediencia, humildad, resignación... ésta es la virtud" (Gómez de Avellaneda, 2001, p. 194). Se sugiere que el papel de "los débiles" corresponde a los esclavos, obligados a aceptar la relativización de los valores éticos y, en particular, de la virtud, debido a su posición de subalternos. Al mismo tiempo, la novela universaliza la problemática social femenina a través del discurso del protagonista afrocubano, al identificarla con la experiencia de la esclavitud (Guerra, 1985, p. 709). Las angustiosas meditaciones sobre la imposibilidad de realizarse como individuo y contribuir a la sociedad en proporción a sus capacidades son aplicables a todos los grupos subalternos sujetos al poder de los grupos dominantes: “¿Sientes la noble ambición de ser útil a tus semejantes y de emplear en el bien general y en tu gloria, las facultades que te oprimen?, pues dóblate bajo su peso y desconócelas, y resígnate a vivir inútil y despreciado, como la planta estéril o como el animal inmundo..." (Gómez de Avellaneda, 2001, p. 193). La propia autora se identifica con el esclavo tanto en la exteriorización de su ansiedad existencial, como en la dimensión subversiva de su discurso (Sommer, 1988, p. 25). Con ello ilustra la tecnología de la dominación, fuente del poder patriarcal, basada en la manipulación de las normas de comportamiento público y privado: “iOh, las mujeres!, ipobres y ciegas víctimas! Como los esclavos, ellas arrastran pacientemente su cadena y bajan la cabeza bajo el yugo de las leyes humanas" (Gómez de Avellaneda, 2001, p. 194).

El poder de la sociedad esclavista está relacionado directamente con su habilidad de tomar en propiedad a seres humanos y mantener control sobre sus vidas y sus cuerpos. En el caso de los esclavos este control se ejerce a través de la violencia física. En el caso de las mujeres libres, según se ve por el fragmento aducido, el control se ejerce indirectamente por medio de la reglamentación de su conducta. El control sobre el cuerpo, según ha señalado Palencia Villa, "ha constituido la obsesión fundamental (en sentido literal), primigenia y vigente sobre la que se sostiene el patriarcado" (Palencia Villa, 2008, p. 41). La sociedad controla a la mujer al obligarla a ajustar su comportamiento de acuerdo a un sistema de valores, en el cual uno de los componentes principales es la asociación entre la virtud femenina y la virginidad. Dentro del contexto religioso:

La mujer debe ser virgen para poder servir a los dioses; el varón sólo debe ser continente durante esos tiempos sagrados. La virginidad está asociada a la condición femenina y apenas es concebible como un atributo masculino" (Martínez Díez, 1991, p. 126). 
Por otra parte, en contexto de la estructura matrimonial el requisito tradicional es que se desea una "virgen de cuerpo y de espíritu" (Martínez Díez, 1991, p. 126). Fuera de la dimensión moral y ética, la virtud femenina tiene un valor económico, y por tanto, el precio que se paga por un cautivo en un sistema esclavista es equivalente a la transacción tradicional que se requiere para efectuar el matrimonio, sea esta una dote que el padre debe entregarle al hombre que se casa con su hija en algunas culturas, o el rescate que el novio está obligado a pagarle a la familia de la mujer en otras. Lo significativo es que la cantidad de la dote o del rescate puede ser negociada en dependencia de las cualidades físicas y del estado de cierta integridad corporal de la mujer.

Gómez de Avellaneda llega a Sevilla con un bagaje emocional adquirido como resultado de una serie de experiencias sociales negativas vividas en Cuba y en España. Siendo hija de una familia de buena posición económica y social dentro de la élite camagüeyana, su primer enfrentamiento con la cultura hegemónica masculina se produjo cuando sus familiares intentaron arreglar un matrimonio de conveniencia en contra de su voluntad. Al llegar al viejo mundo, la joven escritora tiene que sufrir las consecuencias de la avaricia de su padrastro, quien se negaba a entregarle la herencia de su padre. También se expone a las tentativas de ser utilizada como propiedad de la familia por su hermano y tío paterno, deseosos de complacer a sus amigos y formar vínculos de parentesco con personas de cierta influencia social a costa de la voluntad de Gertrudis. La resistencia que la joven despliega en todas estas situaciones moldea su conciencia independiente, y esto a su vez influye en la configuración de las/los protagonistas de sus obras teatrales, y de sus dos primeras novelas, la ya mencionada Sab y Dos mujeres.

Cronológicamente, el estreno de Leoncia coincide con una intensificación del escrutinio público de la imagen femenina, que se produce como consecuencia de la ansiedad que el sistema del poder hegemónico experimenta frente al aumento de la participación de las mujeres en el proceso de la producción cultural, habilitado inicialmente por los avances de la llustración, y luego por el desarrollo del romanticismo. La transformación de la sociedad en la Europa del siglo XVIII:

trajo consigo un cambio de las definiciones de la diferenciación sexual y un nuevo modo de representar y experimentar la subjetividad, y [...] estos cambios abrieron un canal que permitió a las mujeres afirmarse como productoras de cultura impresa" (Kirkpatrick, 1991, p. 12).
Las modificaciones en el modelo de conducta femenina desestabilizan la hegemonía del poder patriarcal, basada tradicionalmente en una estricta diferenciación jerárquica de los rasgos que definían la masculinidad y la feminidad. Las raíces de dicha desestabilización tienen que ver con el discurso moral sobre el amor en el siglo XVIII que busca desplazar "la desconfianza hacia las pasiones como impulsos difícilmente controlables" promovida en la literatura moral y religiosa de la sociedad de Antiguo Régimen, y sustituirla con una valoración positiva de los afectos moderados (Bolufer Peruga, 2011, p. 12). Si en la cultura del Antiguo Régimen el amor se definía en términos de enfermedad, enajenación, arrebato, locura, el discurso ilustrado entreabre la puerta a la posibilidad de una reconciliación entre la pasión amorosa, las inclinaciones naturales del individuo, la armonía familiar, y la utilidad social, todo ello dentro del contexto de una nueva sensibilidad aceptable tanto para las mujeres como para los hombres:

gracias a los recursos textuales que buscan la verosimilitud y la identificación entre el público y los protagonistas, se aspira a que mujeres y hombres se empapen, a través de un proceso de ósmosis, de los sentimiento y virtudes de los personajes, que sufran con sus penas y se complazcan con su felicidad, modelando a imagen y semejanza de los héroes y heroínas de la literatura sus propios deseos y aspiraciones amorosas (Bolufer Peruga, 2011, p. 13).

En el drama Leoncia, los protagonistas expresan sus sentimientos en términos esencialmente similares, ejemplificando el mencionado proceso de ósmosis. Se produce una sensibilización/ feminización del modelo de conducta masculino, es decir, Carlos demuestra su vulnerabilidad emocional, mientras que Leoncia se apropia de las características típicas de la conducta masculina. Por ejemplo, en la escena VII del primer acto, Leoncia le declara a Carlos que le ama "con delirio, con idolatría, cual no ha amado jamás una mujer ninguna," pero renuncia a contarle detalles de su vida. Habla desde una posición de poder, y su lenguaje apasionado desafía las restricciones morales y religiosas impuestas al discurso femenino. La reacción del joven inicialmente es de súplica y luego de subordinación pasiva: "Habla... ¿no ves cuánto sufro? [...] Pues bien: inada te exijo! Esperaré a que quieras abrirme tu corazón; pero el mío es tuyo para siempre [...] antes de conocerte, ime entrego a ti!" (Gómez de Avellaneda, 1917, p. 16).

La creciente preocupación de los hombres con la modificación del papel social de la mujer se pone en evidencia a partir de los años 60 del siglo XVIII, cuan- 
do se hacen más frecuentes las quejas públicas en contra de tales rasgos como una supuesta "marcialidad," "descaro," "despejo" y "conducta licenciosa" atribuidos al comportamiento femenino (Jagoe, 1998, p. 23). En el siglo XIX, el triunfo de los valores éticos y estéticos del movimiento romántico, que en el campo de la cultura literaria resulta beneficioso para las mujeres españolas, obliga al establecimiento patriarcal a cambiar de táctica. En vez de recriminaciones directas en la prensa, se intensifica la promoción cultural del modelo doméstico de subordinación. De ahí que se da un incremento sustancial en el número de publicaciones, de autoría masculina como femenina, enfocadas en la tarea de manipular la conciencia femenina: "sobre todo a partir de 1840 , se publicaron innumerables tratados, artículos, monografías, antologías y discursos describiendo la naturaleza de la mujer y prescribiendo su papel en la sociedad" (Jagoe, 1998, p. 23). La hegemonía patriarcal en la educación y la cultura de España logra imponer cierto control sobre el discurso femenino, y de ahí es que la voz femenina se escinde. Por una parte, el mayor acceso a la tribuna de la cultura impresa abre la puerta al discurso feminista que cuestiona la jerarquía del poder en la sociedad, pero al mismo tiempo, aparecen una serie de voces femeninas que justifican y apoyan dicha hegemonía. Este fenómeno es observable en el discurso periodístico, dirigido "a la mujer tradicional para reforzarle la importancia de su condición de esposa y madre a través del eterno femenino... Se va a potenciar ternura, modestia, humildad y sumisión como ideales sociales y literarios" (Cantizano Márquez, 2004, p. 285). Las publicaciones que enfocan el papel tradicional de la mujer se inscriben en la tradición del discurso moral y edificante y sirven para apuntalar la visión bipolar milenaria de la mujer, es decir, la Santa frente a la Pecadora. En este careo, por supuesto, se privilegia a la primera y se degrada a la segunda. En sus primeros textos literarios, Gertrudis Gómez de Avellaneda, partiendo de una experiencia agudamente personal, cuestiona la dicotomización de las mujeres en dos grupos, el de las que se conforman con el estatus quo y se incorporan en la jerarquía social dentro de en espacio subordinado, y el de las mujeres marginadas por intentar ejercer su propia agencia y llevar un modo de vida independiente.

En el prologo a Leoncia, Emilio Cotarelo sugirió la posibilidad de considerar el asunto del drama en el contexto de una tormentosa relación de amor que la autora tuvo al llegar a Sevilla y el desengaño que sufrió como consecuencia de esta (Gómez de Avellaneda, 1917, p. xi). La relación se mantuvo en se- creto hasta la publicación no autorizada de las cartas privadas de la escritora en 1914 por Lorenzo Cruz de Fuentes. Gómez de Avellaneda sintió una verdadera pasión por el destinatario, mientras que este no le correspondió adecuadamente. Desde la primera epístola se hace evidente la motivación sentimental de la autora, se trata esencialmente de "un acto de seducción: la escritora trata de presentar una imagen de sí misma que agrade al destinatario y capte su deseo" (Kirkpatrick, 1991, p. 134). En las cartas, la dramaturga le cuenta al destinatario noticias sobre el proceso de escritura de su primer drama, luego le comunica su frustración con los ensayos y los actores, y finalmente, concluida la puesta en escena de la obra, le suplica una y otra vez a su amado que venga al estreno. Emilio Cotarelo sugirió que este empeño podía significar que la escritora "fundaba vagas esperanzas de atraerle de nuevo al redil de sus malpagados amores" (Gómez de Avellaneda, 1917, p. 122). Tal vez al insistir en que el destinatario de las cartas asistiera a la función del drama, Gómez de Avellaneda pretendía sensibilizarlo con el cuadro patético de los sufrimientos de sus héroes, esperando lograr el mismo efecto que buscaba provocar en el resto de los espectadores. Más allá de una intencionalidad sentimental privada, se trataba de la misión pública de todo escritor romántico que Jorge Isaacs verbalizó en su novela María: "dulce y triste misión [...] y si suspendéis la lectura para llorar, ese llanto me probará que la he cumplido fielmente" (Isaacs, 1894, p. 7). El destinatario de las cartas, cuya presencia en el estreno fue tan deseada por la dramaturga fue un tal Ignacio de Cepeda, estudiante de leyes, a quien Gertrudis Gómez conoció poco después de su llegada a Sevilla. La cartas de la joven escritora revelan su dilema principal: Ignacio rehusaba formalizar su relación con ella por prejuicios sociales. A pesar de todos los esfuerzos, Gómez de Avellaneda no logró trascender el papel de amante secreta. Para Cepeda, a juzgar por las cartas de Gertrudis, la imagen pública de la joven dramaturga y su modo de vida independiente no encajaban en el modelo de la mujer virtuosa (Gómez de Avellaneda, 1999, p. 31). Parece que Cepeda le recriminaba la falta de virtud, ya que en una carta Gertrudis Gómez le escribe:

"me amaste tu como soy? ¿me crees digna? [...] tú me dices que sea virtuosa; que tú no serás jamás un enemigo de la virtud; que la mía, si la alcanzo, aumentará tu cariño. Amigo mío, yo no soy virtuosa, no; soy una débil criatura, que ha cometido muchas faltas, que se reconoce muy frágil; pero amo a la virtud, la busco, la pido la deseo" (Gómez de Avellaneda, 1999, p. 146). 
En otra carta, Gómez de Avellaneda responde a las incriminaciones de Cepeda con franqueza y claridad:

"¿es tan grave delito amar en una mujer que era libre?", "yo no soy ni monja, ni casada, tu no eres tampoco esclavo de ningún juramento, que te haga un crimen del amor..." (Gómez de Avellaneda, 1999, pp. 160-161).

El empeño de reconfigurar el concepto de la virtud por medio de la retórica de la libertad, de origen ilustrado y romántico, es patente tanto en las cartas privadas dirigidas a Cepeda, como en el discurso dramático de Leoncia.

La clave para entender la visión ética que se ofrece al público en el primer drama de Gómez de Avellaneda, es el contrapunto entre distintas perspectivas sobre la protagonista. La primera perspectiva es falsa, ya que está basada en los rumores sociales que erróneamente le atribuyen a Leoncia una conducta frívola. La segunda es más fiel a la realidad porque está relacionada con la conducta y las cualidades morales de la heroína. La importancia del tema de la virtud se revela desde la primera escena, en la cual se enfoca el conflicto entre las dos caracterizaciones de la protagonista. Por una parte, Carlos destaca las cualidades positivas de la dama, y la describe como un alma noble, pura y superior, mientras que su amigo, don Gaspar, la desvirtúa trayendo a colación una supuesta relación ilícita con el conde Peñaflor, y sugiriendo que esta se basaba en el interés económico:

Don Carlos: ¡Gaspar! Una sola cosa te exijo... ¿Quisiera saber por qué supones que Leoncia es mi dama?

Don Gaspar: Porque lo ha sido del Conde de Peñafiel, como lo sabe todo Madrid. El Conde es anciano, pero es rico; tu, supongo que no te dará tu padre muchos doblones para satisfacer los caprichos de tu hermosa; pero eres joven y amable. No veo, por tanto, un gran inconveniente en que seas actualmente, cerca de Leoncia, lo que fue el Conde antes que tú. (Gómez de Avellaneda, 1917, pp. 4-5).

El comentario sobre la frivolidad de Leoncia no tiene otro fundamento que la afirmación rotunda "lo sabe todo Madrid," lo cual pone en carne viva la problemática situación de la mujer en una sociedad, que controla la percepción pública del concepto de honor y lo instrumentaliza para disciplinar y castigar. La hipocresía de la sociedad patriarcal se confirma con otro comentario de don Gaspar, ya que la falta de virtud se tolera si se mantienen las apariencias: "toda la diferencia consiste en que se hacen ahora las cosas con más reserva y prudencia... Ya vez tú: la sociedad es, a veces, indulgente con las flaquezas; pero siempre condena el descaro, la imprudencia..." (Gómez de Avellaneda, 1917, p. 7).

Carlos manifiesta su indignación al escuchar la alusión a una supuesta frivolidad de Leoncia. Tal vez Gómez de Avellaneda hubiera querido que Cepeda reaccionara del mismo modo ante quienes rumoreaban en su contra, y por boca de Carlos declara que "... el honor de una mujer es sagrado, porque es un ser débil, indefenso..." (Gómez de Avellaneda, 1917, p. 6). Lo curioso es que el propio Carlos, aunque reconoce los defectos de la sociedad y su código de hipocresía, y aun cuando reconoce que el nombre de la dama de su corazón "i... es un nombre mancillado por la calumnia!" (Gómez de Avellaneda, 1917, p. 7) también condiciona la aceptación de Leoncia como esposa con la necesidad de averiguar "si es tan noble y tan pura como mi corazón la desea y la adivina" (Gómez de Avellaneda, 1917, p. 11).

El ideal tradicional de la virtud femenina es representado por Elena definida como "un ángel de hermosura y de inocencia," una "niña" de "puro y tierno corazón" con candor y "casi infantil jovialidad" (Gómez de Avellaneda, 1917, pp. 3-4). El retrato de esta "niña inocente" contrasta con la imagen de Leoncia, que según Carlos es una mujer:

cuya alma de fuego haya marchitado prematuramente en su frente la flor de la juventud. Una cuya mirada de pasión sea un dardo encendido que se clave en la mitad de mi alma; porque sus ojos omnipotentes todo lo traspasen, todo lo venzan. Una cuyo acento vibrante salga de su corazón impregnado de amor para ir a encender el mío... (Gómez de Avellaneda, 1917, p. 4).

Es legítimo suponer que Gertrudis aspiraba a hacerle llegar a Ignacio el mensaje codificado de su alma apasionada, y trataba de hacerle ver su virtud intrínseca por medio del uso de la retórica romántica en Leoncia, y la dificultad de conseguirlo no estaba únicamente relacionada con un posible defecto moral del destinatario, sino con la problemática "posición del sujeto femenino en relación a la elaboración romántica de un lenguaje de subjetividad" (Kirkpatrick, 1991, p. 20):

por una parte, el nuevo movimiento estético parecía fomentar la participación de las mujeres mediante la revalorización del sentimiento y de la individualidad, pero por otra parte, las mujeres encontraban difícil asumir los muchos atributos de la individualidad ro- 
mántica que estaban en conflicto con la norma que relacionaba la identidad femenina con la falta de deseo (Kirkpatrick, 1991, p. 20).

En efecto, el acceso a la producción de la cultura literaria les permitía a las mujeres influir en la transformación de la sociedad porque les daba la oportunidad de cuestionar los valores éticos y estéticos del patriarcado desde una postura protegida por el ideario romántico. Gómez de Avellaneda participa en este proceso promoviendo una visión de la mujer como sujeto y agente de la acción (Guerra, 1985, p. 708). Critica la función atribuida a la mujer como objeto de consumo masculino divulgada a mediados del siglo XVIII por J.J. Rousseau en su apología de la desigualdad en la educación masculina y femenina titulada Emilio o de la educación:

uno debe ser activo y fuerte, el otro pasivo y débil: es totalmente necesario que uno quiera y pueda; basta que el otro se resista un poco. Establecido este principio de él se sigue que la mujer está hecha especialmente para agradar al hombre (Rousseau, 1997, p. 535).

En Leoncia, Gómez de Avellaneda le permite a la protagonista asumir un papel activo en la búsqueda romántica del bello ideal y llega a representar una subjetividad femenina en términos que desafían las limitaciones impuestas por el discurso masculino. Procede del mismo modo que en su discurso privado epistolar y en varios otros textos narrativos y dramáti- cos que publica posteriormente. Cabe señalar que el rechazo de la relativización tradicional del concepto de la virtud se realiza desde una postura que asocia este último con las cualidades intrínsecas del ser humano y no con la función que le ha sido impuesta al individuo por la estructura social. Tal es el caso del protagonista esclavo, que como ya señalamos anteriormente, metaforiza la experiencia femenina. En este proceso de apropiación de un lenguaje de poder, como ha explicado Torres-Pou, el discurso narrativo y epistolar de Gómez de Avellaneda inevitablemente se contamina por la retórica de la misma tradición patriarcal contra la cual levanta la voz (Torres-Pou, 1993, p. 58). Sin embargo, conviene subrayar que esto no constituye un demérito, ya que le permite a la autora establecer un canal de comunicación con el público amamantado con los conceptos provenientes de dicha tradición. De otro modo a la autora le resultaría mucho más difícil, si no imposible, transmitir una nueva visión crítica que estaba en conflicto con la ideología hegemónica. El análisis temático de Leoncia que ensayamos en las páginas precedentes en el contexto de los primeros textos narrativos y epistolares de Gertrudis Gómez de Avellaneda, demuestra una profunda falta de conformidad, por parte de la escritora, con las técnicas de manipulación del concepto de la virtud, y su firme voluntad por reconfigurar el paradigma cultural jerárquico prevalente en la sociedad española de su época.

\section{BIBLIOGRAFÍA}

Bolufer Peruga, M. (2011). 'Hombres de bien': modelos de masculinidad y expectativas femeninas, entre la ficción y la realidad. Cuadernos de llustración y Romanticismo, 15, pp. 7-31.

Cantizano Márquez, B. (2004). La mujer en la prensa femenina del XIX. Ambitos: Revista internacional de comunicación, 11, p.15.

Cotarelo y Mori, E. (1930). La Avellaneda y sus obras: ensayo biográfico y crítico. Madrid: Tipografía de Archivos.

Espronceda, J. (1978). El estudiante de Salamanca; El diablo mundo. Madrid: Castalia.

Gies, D. (1996). El teatro en la España del siglo XIX. Cambridge: Cambridge University Press.

Gómez de Avellaneda, G. (1917). Leoncia. Madrid: Tipografía de la Revista de Archivos, Bibliotecas y Museos.
Gómez de Avellaneda, G. (1990). Obra selecta. Caracas: Biblioteca Ayacucho.

Gómez de Avellaneda, G. (1999). Autobiografía y epistolarios de amor. Newark: Juan de la Cuesta.

Gómez de Avellaneda (2001). Sab. Manchester: Manchester University Press.

Guerra, L. (1985). Estrategias femeninas en la elaboración del sujeto romántico en la obra de Gertrudis Gómez de Avellaneda. Revista iberoamericana, LI, 132-133, pp. 707-722. http://dx.doi. org/10.5195/reviberoamer.1985.4094

Isaacs, J. (1894). María: novela americana. París: Librería Garnier hermanos.

Jagoe, C. (1998). La misión de la mujer. En: Jagoe, C. (ed.). La mujer en los discursos de género: textos y contextos en el siglo XIX. Barcelona: Icaria, pp. 21-53.
Kirkpatrick, S. (1991). Las románticas: escritoras y subjetividad en España, 18351850. Madrid: Cátedra.

Martínez Díez, F. (1991). Domingo de Guzmán: evangelio viviente. Salamanca: San Esteban.

Palencia Villa, R. M. (2008). Recuperar el propio cuerpo: discursos fílmicos de resistencia. En: Clúa Ginés, I., Pitarch, P. (eds.). Pasen y vean. Estudios culturales. Barcelona: Universitat Oberta de Catalunya , pp. 41-46.

Piñeyro, E. (1904). Gertrudis Gómez de Avellaneda. Bulletin Hispanique, 6, 2, pp. 143-156. http://dx.doi.org/10.3406/ hispa.1904.1403

Prado Mas, M. (2004). El teatro de Gertrudis Gómez de Avellaneda. [Tesis doctoral]. Universidad Complutense de Madrid: Madrid. Recuperado de http://eprints. ucm.es/tesis/fll/ucm-t25107.pdf 
Rousseau, J.J (1997). Emilio o de la educación. Madrid: Alianza.

Selimov, A. (1999). La verdad vence apariencias: hacia la ética de Gertrudis Gómez de Avellaneda a través de su prosa. Hispanic review, 67, 2, pp. 215-241. http://dx.doi. org/10.2307/474915
Sommer, D. (1988). Sab c'est moi. Genders, 2, pp. 111-126.

Torres-Pou, J. (1993). La ambigüedad del mensaje feminista de 'Sab' de Gertrudis Gómez de Avellaneda. Letras Femeninas, 19, 1/2, pp. 55-64. 